\title{
Pile Driving Vibration Attenuation Relationships: Overview and Calibration Using Field Measurements
}

\author{
Athina Grizi, A.M.ASCE ${ }^{1}$, Adda Athanasopoulos-Zekkos, M.ASCE ${ }^{2}$ and \\ Richard D. Woods, Dist.M.ASCE ${ }^{3}$
}

${ }^{1}$ Assistant Professor, Department of Civil Engineering, University of Nottingham, University Park, Nottingham, NG7 2RD; e-mail: a.gkrizi@ nottingham.ac.uk

${ }^{2}$ Associate Professor, Department of Civil and Environmental Engineering, University of Michigan, 2350 Hayward St., Ann Arbor, MI 48109; e-mail: addazekk@umich.edu

${ }^{3}$ Professor Emeritus, Department of Civil and Environmental Engineering, University of Michigan, 2350 Hayward St., Ann Arbor, MI 48109; e-mail: rdw@umich.edu

\begin{abstract}
Empirical equations to predict ground motions during construction operations have been suggested by various researchers. These empirical correlations were developed after measuring particle velocities on the ground surface during pile installation or other construction activities. When waves travel through the soil and away from the impact source, energy is lost due to material and geometric damping, respectively. This paper presents an overview of existing wave attenuation formulas for man-made induced vibrations. The authors collected ground motion measurements from impact pile driving by installing sensors at varying depths and very close to driven piles. A conventional line array of surface sensors was also used. Attenuation curves developed from records of both surface and in-depth sensors are presented.
\end{abstract}

\section{INTRODUCTION}

Ground vibrations caused by pile driving can potentially damage nearby structures and underground utilities. It is therefore important to predict ground motions, usually as peak particle velocities, that are generated by pile driving activities. While the interaction of soil and pile is difficult to evaluate in layered soil with large vibratory strains that are developed in the near field, the effect of pile hammer and pile type in creating vibrations is somewhat easier to analyze. Vibration attenuation is described with distance decay plots or scaled-distance plots. Scaling of distance is commonly used to predict peak particle velocities in blasting operations, and the square root scaling was adopted for pile driving activities; distance is divided by the square root of the hammer energy (Dowding 1996).

The first part of this paper provides a review of published models that predict peak particle velocities due to construction operations. All the expressions were developed by monitoring ground motions on the earth surface. In this study, ground motions were measured during impact pile driving with accelerometers and geophones pushed into different soil layers starting very close to the pile. Surface ground motions were also recorded with a line array of geophones. Attenuation characteristics were investigated by fitting published decay expressions through the recorded data. The same H-pile size and a hammer with a similar rated energy were used at both sites presented, and attenuation coefficients for both a loose sandy soil and a hard sandy clay are determined. 


\section{VIBRATION ATTENUATION MODELS}

Many researchers have recorded ground surface vibrations during construction operations. Two categories of empirical models were developed to predict ground motions: (1) attenuation equations with distance from the source and (2) attenuation equations with scaled-distance from the source. It is the horizontal distance from the source to the measuring point that is usually taken as the distance in these relations. However, when a source of vibration other than a point source, like a pile, is generating waves through the soil, the actual distance from the measuring point to the source point may not be the horizontal.

\section{1) Vibration Attenuation with Distance from the source}

One of the earliest empirical correlations was presented by Bornitz (1931) and is referred to here as the Bornitz equation:

$$
v_{2}=v_{1}\left(\frac{r_{1}}{r_{2}}\right)^{n} \exp \left[-\alpha\left(r_{2}-r_{1}\right)\right] \quad \text { Eq. } 1
$$

where $V_{1}$ and $V_{2}$ are the vibration amplitudes at distance $r_{1}$ and $r_{2}$ from the source, $n$ is a coefficient depending on the wave type and $\alpha$ is the attenuation coefficient (units of 1/distance). This formula combines two components of attenuation: geometric damping with distance from the source, represented by the first part of the equation, and material damping as waves travel through the soil, provided by the exponential part of the equation. Geometric damping coefficients, $n$, were analytically determined by Gutowski and Dym (1976) and are shown in Table 1.

Table 1. Geometric attenuation coefficients (after Gutowski and Dym 1976)

\begin{tabular}{cccc}
\hline Source Type & Wave & Source Location & n \\
\hline Line & Rayleigh & Surface & 0 \\
Line & Body & Surface & 1.0 \\
Point & Rayleigh & Surface & 0.5 \\
Point & Body & Surface & 2.0 \\
Buried Line & Body & Interior & 0.5 \\
Buried Point & Body & Interior & 1.0 \\
\hline
\end{tabular}

The material damping coefficient, $\alpha$, is frequency dependent. Table 2 was prepared with data collected for twenty years from 36 sites by Woods and Jedele (1985) and presents values of the attenuation coefficient, $\alpha$, for frequencies of 5 and $50 \mathrm{~Hz}$ and different classes of soil. Vibrations tend to drop off more rapidly with distance through softer materials. Alpha values for different frequencies can be estimated from $\alpha_{2}=\alpha_{1}\left(f_{2} / f_{1}\right)$, where $\alpha_{1}$ is the known attenuation coefficient at frequency $f_{1}$ and $\alpha_{2}$ is the unknown attenuation coefficient at frequency $f_{2}$. It should be noted that the Bornitz formulation was proposed for small amplitude motion at long distance from an earthquake. However, based on the recorded data presented here, it is a very good approximate model near the source of high amplitude motion as well. 
Wiss (1981) suggested a pseudo-attenuation formula with distance, which is essentially a best-fit power equation as:

$$
v=k D^{n} \quad \text { Eq. } 2
$$

where $v$ is the peak particle velocity, $k$ is the value of the velocity at one unit of distance, $D$ is the distance from the vibration source and $n$ is the slope or attenuation rate. The pseudo-attenuation coefficient, $n$, accounts for both geometric and material damping but this simplified relationship tends to overpredict ground motions at very large and very small distances.

The California Department of Transportation indicated that pile driving vibrations appear to decay differently than the Rayleigh waves, probably due to the presence of a significant proportion of body waves (Hendriks 2004). The following attenuation equation was suggested for pile driving operations:

$$
v_{2}=v_{1}\left(\frac{r_{1}}{r_{2}}\right)^{\beta}
$$

where $V_{1}$ and $V_{2}$ are the vibration amplitudes at distance $r_{1}$ and $r_{2}$ from the source and $\beta$ is a dimensionless attenuation parameter, independent of frequency.

\begin{tabular}{|c|c|c|c|}
\hline \multirow[t]{2}{*}{ Class } & \multicolumn{2}{|c|}{$\begin{array}{c}\text { Attenuation } \\
\text { Coefficient, } \alpha(1 / \mathrm{m})\end{array}$} & \multirow[t]{2}{*}{ Description of material ( $\mathbf{N}=$ SPT Blow Count) } \\
\hline & $5 \mathbf{H z}$ & $50 \mathrm{~Hz}$ & \\
\hline $\begin{array}{c}\mathrm{I} \\
(\mathbf{N}<\mathbf{5})\end{array}$ & $\begin{array}{c}0.01 \text { to } \\
0.033\end{array}$ & $\begin{array}{c}0.1 \text { to } \\
0.33\end{array}$ & $\begin{array}{l}\text { Weak or soft soils (shovel penetrates easily): lossy soils, dry or } \\
\text { partially saturated peat and muck, mud, loose beach sand and dune } \\
\text { sand, recently plowed ground, soft spongy forest or jungle floor, } \\
\text { organic soils, topsoil. }\end{array}$ \\
\hline $\begin{array}{c}\text { II } \\
(5<\mathrm{N}<15)\end{array}$ & $\begin{array}{c}0.0033 \text { to } \\
0.01\end{array}$ & $\begin{array}{c}0.033 \text { to } \\
0.1\end{array}$ & $\begin{array}{l}\text { Competent soils (can dig with shovel): most sands, sandy clays, } \\
\text { gravel, silts, weathered rock. }\end{array}$ \\
\hline $\begin{array}{l}\text { III } \\
(\mathbf{1 5}<\mathrm{N}<\mathbf{5 0})\end{array}$ & $\begin{array}{l}0.00033 \\
\text { to } 0.0033\end{array}$ & $\begin{array}{l}0.0033 \\
\text { to } 0.033\end{array}$ & $\begin{array}{l}\text { Hard soils (cannot dig with shovel, need pick to break up): dense } \\
\text { compacted sand, dry consolidated clay, consolidated glacial till, } \\
\text { some exposed rock. }\end{array}$ \\
\hline $\begin{array}{c}\text { IV } \\
(\mathbf{N}>\mathbf{5 0})\end{array}$ & $<0.00033$ & $<0.0033$ & $\begin{array}{l}\text { Hard competent rock (difficult to break with hammer): bedrock, } \\
\text { freshly exposed hard rock. }\end{array}$ \\
\hline
\end{tabular}

Table 2. Proposed classification of earth materials by attenuation coefficient (after Woods and Jedele 1985)

\section{2) Vibration Attenuation with Scaled-Distance from the source}

Heckman and Hagerty (1978) investigated pile driving cases and modified a scaleddistance relationship proposed by Wiss (1967) and Attewell and Farmer (1973):

$$
v=K\left(\frac{D}{\sqrt{E}}\right)^{-1}
$$

Eq. 4

where $V$ is the peak particle velocity, $K$ is a factor dependent on pile impedance, $D$ is the distance from the vibration source and $E$ is the impact energy transferred from the hammer to the pile. The 
ground vibrations and the $K$ factor increase with decreasing pile impedance. The pile impedance, $I$, is defined as the product of the pile section area, $A$, times the pile density, $\rho$, times the wave speed in the pile material, $c(I=A \rho c)$. Attewell and Farmer (1973) proposed a conservative value of $K=1.5$ for Eq. 3 .

Wiss (1981) also suggested that it is convenient to have the distance and the energy from the source in a single expression:

$$
v=k\left(\frac{D}{\sqrt{E}}\right)^{-n}
$$

where $v$ is the peak particle velocity, $k$ is the value of velocity at $\mathrm{D} / \sqrt{\mathrm{E}}=1, D$ is the distance from the vibration source, $E$ is the impact energy transferred from the hammer to the pile an $n$ is the slope or attenuation rate.

Attewell et al. (1992a, 1992b) suggested that a quadratic regression curve rather than a linear regression curve provides a better prediction model. A statistical analysis of impact pile driving vibration data was performed in order to get the mean (Best Fit), one-half standard deviation (SD) and one standard deviation line:

$$
\begin{array}{lc}
\log v=-0.519+1.38 \log \left(\frac{\sqrt{E}}{D}\right)-0.234 \log ^{2}\left(\frac{\sqrt{E}}{D}\right) & \begin{array}{c}
\text { Eq. 6a } \\
\text { Best Fit }
\end{array} \\
\log v=-0.296+1.38 \log \left(\frac{\sqrt{E}}{D}\right)-0.234 \log ^{2}\left(\frac{\sqrt{E}}{D}\right) & \begin{array}{c}
\text { Eq. 6b } \\
\text { (One-half SD) }
\end{array} \\
\log v=-0.073+1.38 \log \left(\frac{\sqrt{E}}{D}\right)-0.234 \log ^{2}\left(\frac{\sqrt{E}}{D}\right) & \begin{array}{c}
\text { Eq. 6c } \\
\text { (SD) }
\end{array}
\end{array}
$$

where $v$ is the peak particle velocity, $D$ is the distance from the vibration source, $E$ is the impact energy transferred from the hammer to the pile. A family of curves for the prediction of particle velocities at distances of up to $20 \mathrm{~m}$ were determined.

\section{DESCRIPTION OF MONITORED SITES}

The authors obtained ground vibration data during impact pile driving from five sites located in Michigan. In this paper, results from two sites are presented; site M-139 and site US131. Steel H-piles $(360 \times 109 \mathrm{~mm} \times \mathrm{kg} / \mathrm{m})$ were driven using diesel hammers. A Pileco D30-32 hammer with a rated energy of 94,800 Joules was used for site M-139 and a Delmag D30-32 hammer with a rated energy of 103,000 Joules was used for site US-131. Subsurface conditions at site M-139 consisted of loose sands to a depth of $9 \mathrm{~m}$ underlain by layers of medium dense sands to a depth of about $18 \mathrm{~m}$. The water table was encountered at $1.6 \mathrm{~m}$ below the ground surface. The soil profile of site US-131 consisted of about $6 \mathrm{~m}$ of medium dense sand over a hard sandy clay to a depth of $12 \mathrm{~m}$ and a dense sand below that. The water table was about $1 \mathrm{~m}$ below the surface.

One of the unique aspects of this work was the installation of sensors in the ground starting very close to the pile $(0.15 \mathrm{~m})$ and at different radial distances. Triaxial accelerometers were pushed to a depth of $7.8 \mathrm{~m}$ into a loose to medium dense sand at site M-139. Triaxial 
accelerometers and geophones were successfully installed to a depth of $10.8 \mathrm{~m}$ into a hard sandy clay layer at site US-131. A conventional line array of sensors was also placed on the ground surface at both sites. The maximum energy transferred to the top of the pile (EMX) was determined using the Pile Driver Analyzer (PDA) system by Pile Dynamics, Inc.. Ground motion measurements were recorded simultaneously from the embedded and surface sensors for the whole duration of pile driving; a sampling rate of $1 \mathrm{kHz}$ was used. In this paper, vertical peak particle velocities (PPV) recorded only when the pile tip was approaching the sensors' elevation will be presented and their attenuation characteristics will be analyzed using the decay equations discussed earlier. It is important to note that the energy transferred to the pile and the actual distance from the source (pile tip) to the sensor location will be used as input in all the formulas. Table 3 presents a summary of the piles, hammers, energies and soil layers that attenuation parameters will be derived for the two sites. It is obvious that the only difference between the tested sites is the type of soil, thus investigation of differences in the attenuation coefficients will be explored. More details about the field testing procedures can be found in Athanasopoulos-Zekkos et al. (2013) and Gkrizi (2017).

Table 3. Site Information Summary

\begin{tabular}{cccccc}
\hline Site & Pile & Hammer & Rated Energy $(\mathbf{J})$ & EMX $(\mathbf{J})$ & Soil \\
\hline M-139 & HP 360x109 & Pileco D30-32 & 94,800 & 47,000 & Loose sand \\
US-131 & HP 360x109 & Delmag D30-32 & 130,000 & 44,000 & Sandy clay \\
\hline
\end{tabular}

\section{GROUND VIBRATION DATA AND COMPARISON OF CORRELATIONS}

\section{M-139 SITE - Loose Sand layer}

Vibration measurements were performed using triaxial accelerometers located at $0.2,0.8$ and $2 \mathrm{~m}$ horizontally from the pile, and buried at a depth of $7.8 \mathrm{~m}$. Geophones were also placed on the ground surface at a distance of 2, 5, 10.5 and $12.3 \mathrm{~m}$ from the pile. Figure 1a presents peak particle velocities versus distance recorded from the buried sensors when the pile tip was being driven from $6.3 \mathrm{~m}$ to $8 \mathrm{~m}$; this depth range was chosen because it brackets the location of the sensors. Coefficients of attenuation were determined by fitting Eqs. 1 to 3 to the recorded data. The three prediction models appear to provide a good correlation as denoted by the coefficient of determination $\left(\mathrm{R}^{2}\right)$. In addition, the attenuation rates $(n=0.72$ and $\beta=0.68)$ derived by Eq. 2 and Eq. 3 are found to be very close. Figure $1 b$ shows the same data plotted versus the scaled-distance and a comparison of Eqs. 4 to 6 that follow the scaled-distance approach is made. The best fit line proposed by Attewell et al. (1992) underestimates the peak particle velocities, while the standard deviation line seems to overestimate the recorded amplitudes. The attenuation coefficient, $\mathrm{n}=0.72$, found by the Wiss (1981) formula (Eq. 5), is the same as the Wiss (1981) pseudo-attenuation formula with distance (Eq. 2).

Figures $2 \mathrm{a}$ and $2 \mathrm{~b}$ depict recorded data by the surface geophones versus distance and scaled-distance, respectively when the pile tip was being driven from $6.3 \mathrm{~m}$ to $8 \mathrm{~m}$. The comparison shows that the correlations with distance (Fig. 2a) are good. The same attenuation coefficient, $\alpha=0.2$, was found as for the buried sensors. This indicates that the same $\alpha$ coefficient can be used to fit both near field (buried sensors) and far field (surface sensors) data if the Bornitz equation is to be used. However, the calculated attenuation rates $n$ and $\beta$, are higher than those derived from 
the buried sensors' data. For the scaled-distance approach, the Heckman and Hagerty (1978) correlation does not provide a good prediction for particle velocities, while the Wiss (1981) relationship is a good fit. The Attewell et al. (1992) one and one half standard deviation lines overestimate the particle velocities.

An attempt was made to combine the near field (buried sensors) and the far field data (surface sensors) and fit the empirical models to see if a good correlation could be derived with any of the models. Figure 3 a presents the attenuation models with distance from the source. The solid red line is the Bornitz fit having as reference point the sensor that is closest to the pile $(0.2$ $\mathrm{m})$ with an attenuation coefficient $\alpha=0.1$. This correlation appears to match the data closely, with an exception of the amplitudes recorded by the surface geophone closest to the pile $(2 \mathrm{~m})$, which fall above the line. If the Bornitz equation is fitted through the data with a reference point the surface geophone closest to the pile (dashed red line), the PPVs are slightly overpredicted. The Hendriks (2004) correlation overestimates the PPVs for large distances from the pile, while the Wiss (1981) power equation suggests a better prediction, but would overpredict vibration amplitudes for distances larger than $10 \mathrm{~m}$. Figure $3 \mathrm{~b}$ shows a comparison of the empirical models with scaled-distance. The Heckman and Hagerty (1978) approach suggests a weak correlation as almost all data fall above the line. The Attewell et al. (1992) formula is more conservative, overestimating the PPVs.

\section{US-131 SITE - Sandy Clay layer}

Vibration monitoring was conducted using accelerometers and geophones at $0.15,0.8,2 \mathrm{~m}$ from the pile, buried at a depth of $10.8 \mathrm{~m}$. Geophones were placed on the ground surface at distances 2, 4.6, 7.6, 10.7 and $19.8 \mathrm{~m}$ from the pile. Figure 4a depicts PPVs versus distance recorded from the buried sensors when the pile tip was being driven from 9.5 to $11 \mathrm{~m}$; just above the sensors' depth. Again, the three attenuation formulas with distance (Eqs. 1 to 3 ) provide a good fit. Note that there is an increase in attenuation rate $(\alpha=0.6$ and $\beta=0.93)$, which is something expected since vibrations in clays tend to drop off more rapidly than those in sands. Figure $4 \mathrm{~b}$ presents the same data versus scaled-distance and a comparison of Eqs. 4 to 6 fitted through the amplitudes. The Heckman and Hagerty (1978) and Attewell et al. (1992) models underestimate the PPVs.

Figures $5 \mathrm{a}$ and $5 \mathrm{~b}$ show recorded data by the surface geophones versus distance and scaleddistance, respectively when the pile tip was being driven from $9.5 \mathrm{~m}$ to $11 \mathrm{~m}$. The Bornitz equation with $\alpha=0.2$ suggests to be the best prediction, even if it is on the conservative side. In general, the Heckman and Hagerty (1978) and the Attewell et al. (1992) formulas overestimate the PPVs, while the correlations proposed by Wiss (1981) and Hendriks (2004) decay very fast and underestimate amplitudes recorded by the furthest geophone from the pile $(19.8 \mathrm{~m})$.

Figure 6a presents recorded data from both the near field and far field and a comparison of the attenuation models with distance from the source. It is of interest to note that the same 

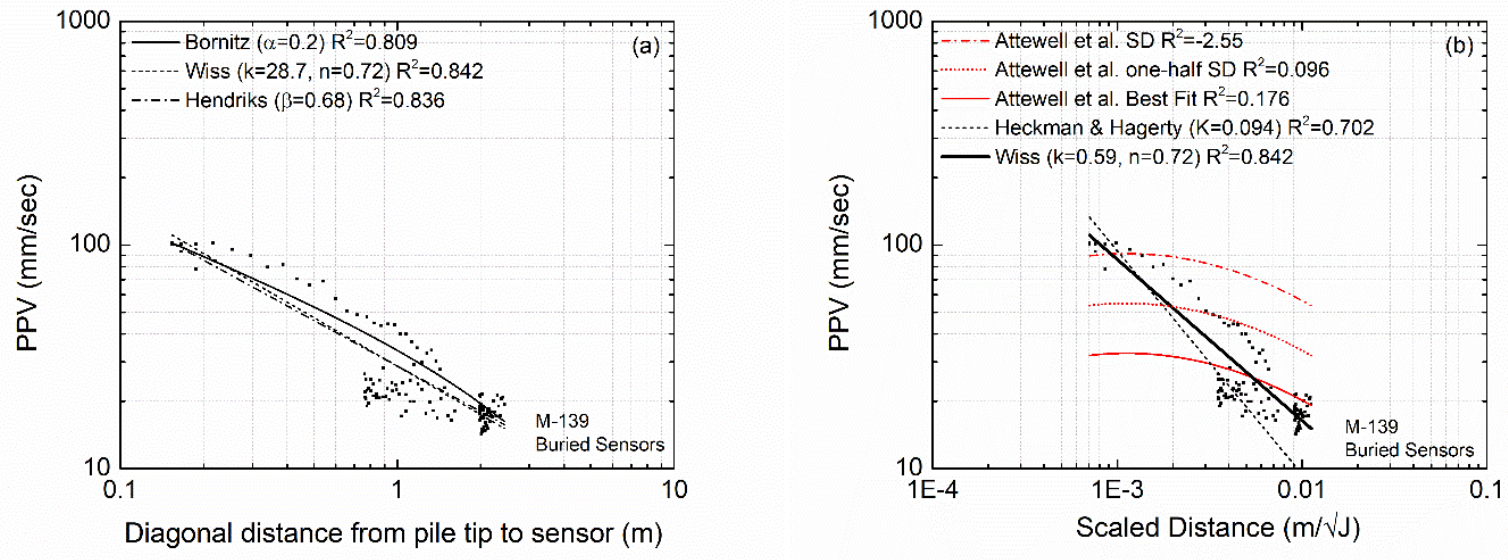

Figure 1. Attenuation of PPV for buried sensors at M-139 site with (a) distance; and (b) scaled-distance
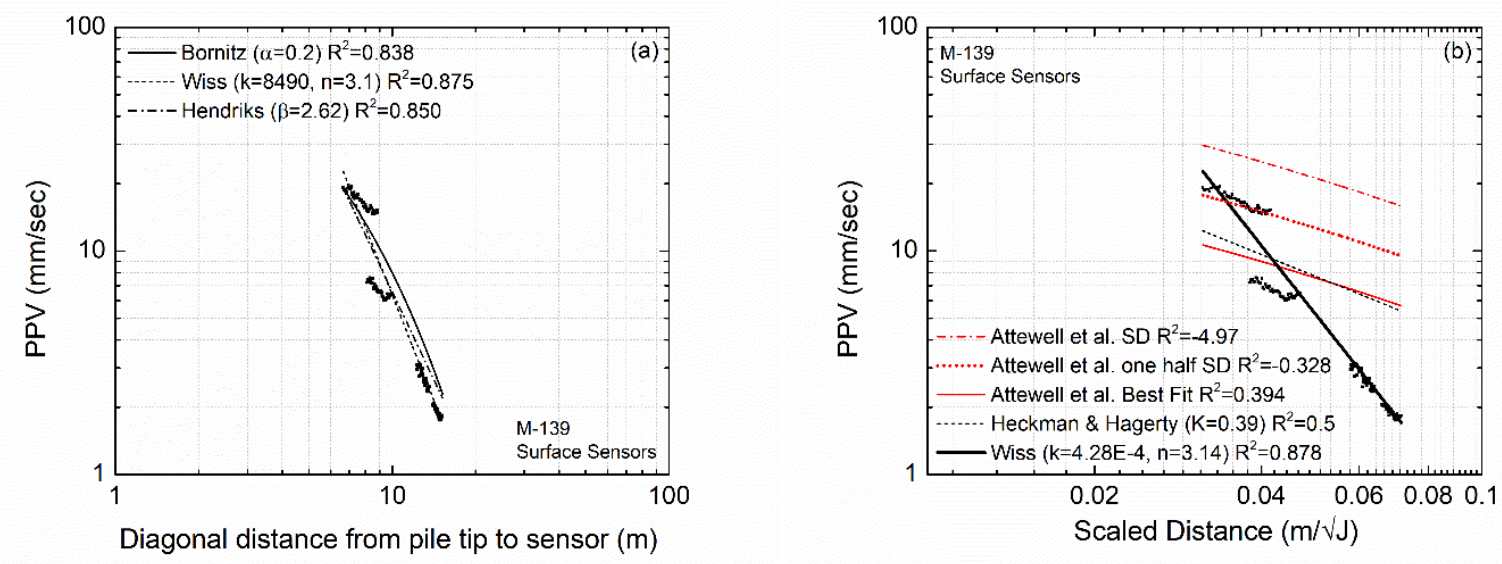

Figure 2. Attenuation of PPV for surface sensors at M-139 site with (a) distance; and (b) scaled-distance
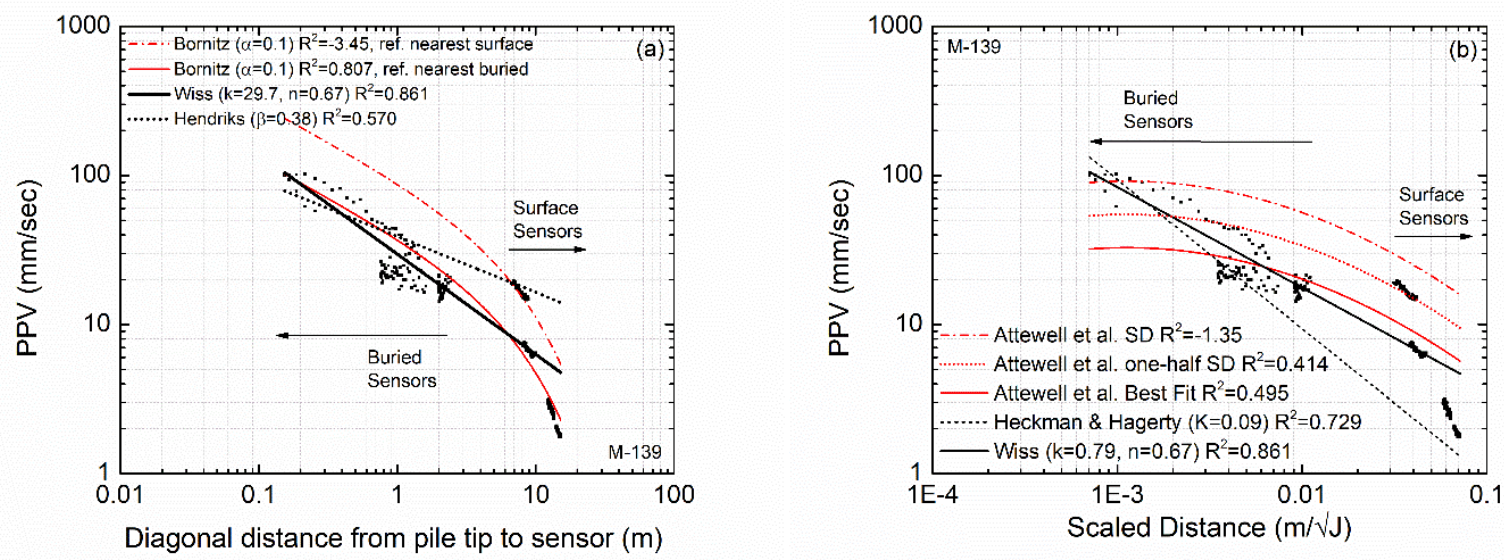

Figure 3. Attenuation of PPV for all sensors at M-139 site with (a) distance; and (b) scaleddistance 

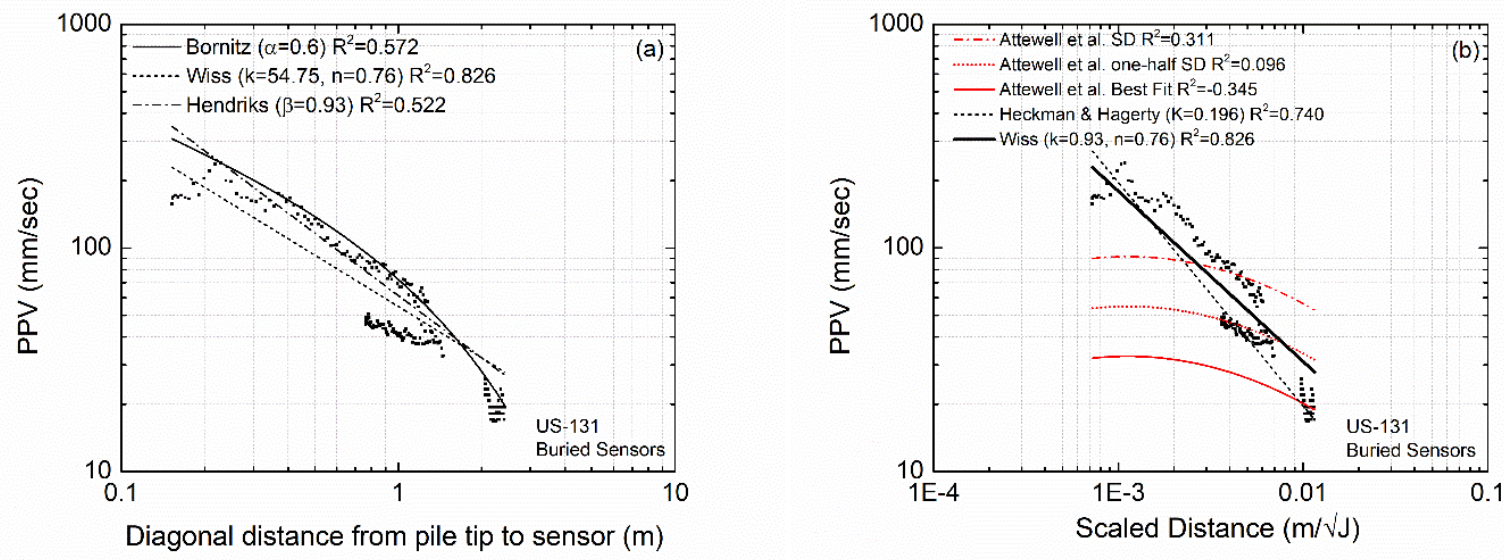

Figure 4. Attenuation of PPV for buried sensors at US-131 site with (a) distance; and (b) scaled-distance
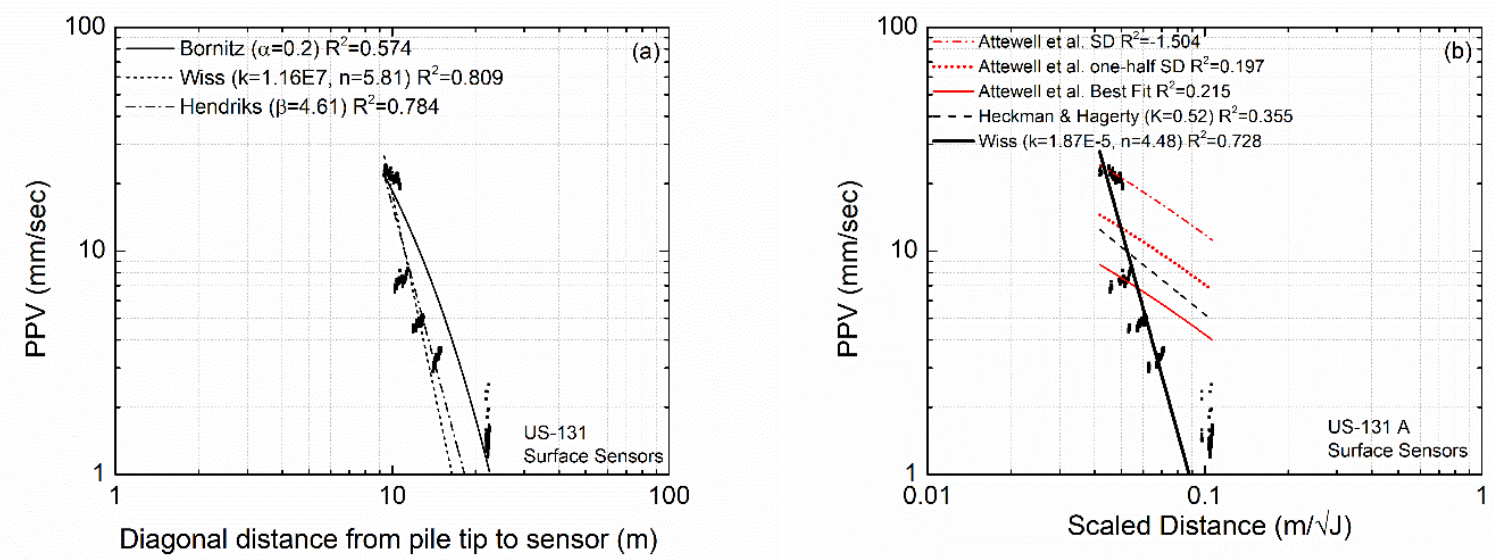

Figure 5. Attenuation of PPV for surface sensors at US-131 site with (a) distance; and (b) scaled-distance
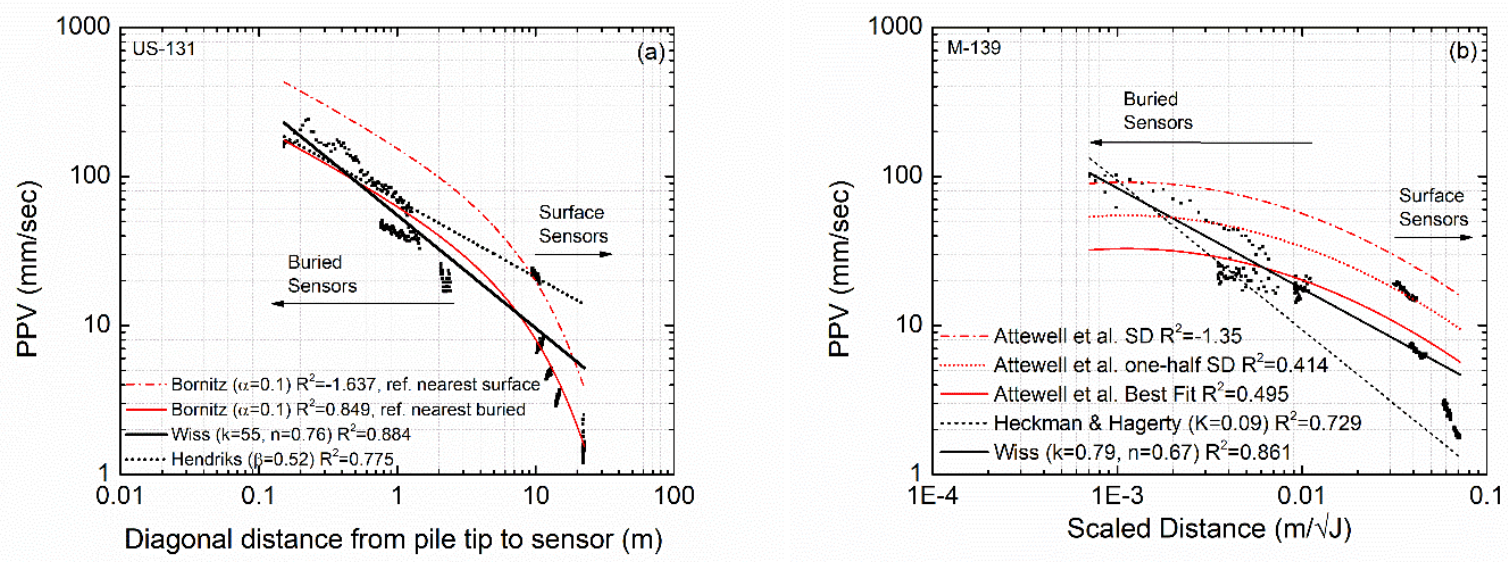

Figure 6. Attenuation of PPV for all sensors at US-131 site with (a) distance; and (b) scaleddistance 
attenuation coefficient $\alpha=0.1$ was found when the Bornitz equation was fitted through the data as in M-139 site. As with the sand layer (Fig. 3a), the Bornitz formula matches the data closely (solid red line with reference point the sensor closest to the pile at $0.15 \mathrm{~m}$ ) with an exception of the amplitudes recorded by the surface geophone closest to the pile $(2 \mathrm{~m})$ which fall above the line. However, using the surface geophone closest to the pile as the reference point (red dashed line) would slightly overestimate the amplitudes, but would still be a very good approximate model for attenuations of vibrations from pile driving. Woods and Jedele (1985) reported similar $\alpha$ coefficients for these classes of soils (Table 2) and a frequency of $50 \mathrm{~Hz}$, which is typical for pile driving operations. All the other models would underestimate or overestimate the velocity amplitudes. Also, the attenuation parameters (n and $\beta$ ) are slightly higher than those found for the sandy layer. Table 4 shows a summary of the calculated attenuation parameters for both sites.

Table 4. Attenuation parameters derived for sites M-139 and US-131

\begin{tabular}{|c|c|c|c|c|c|c|c|c|}
\hline \multirow[t]{2}{*}{ Site } & & \multirow{2}{*}{$\frac{\text { Bornitz }}{\alpha(1 / \mathrm{m})}$} & \multicolumn{2}{|c|}{ Wiss } & \multirow{2}{*}{$\begin{array}{c}\text { Hendricks } \\
\beta\end{array}$} & \multirow{2}{*}{$\begin{array}{c}\begin{array}{c}\text { Heckman } \\
\text { \& Hagerty }\end{array} \\
\text { K }\end{array}$} & \multicolumn{2}{|c|}{$\begin{array}{c}\text { Wiss Scaled } \\
\text { Distance }\end{array}$} \\
\hline & & & $\mathbf{k}$ & $\bar{n}$ & & & $\mathbf{k}$ & $\mathbf{n}$ \\
\hline \multirow[t]{4}{*}{ M-139 } & Buried & 0.2 & 28.7 & 0.72 & 0.68 & 0.094 & 0.59 & 0.72 \\
\hline & $\mathrm{R}^{2}$ & 0.809 & \multicolumn{2}{|c|}{0.842} & 0.836 & 0.702 & \multicolumn{2}{|c|}{0.842} \\
\hline & Surface & 0.2 & 8490 & 3.1 & 2.62 & 0.39 & $4.28 \mathrm{E}-4$ & 3.14 \\
\hline & $\mathrm{R}^{2}$ & 0.838 & \multicolumn{2}{|c|}{0.875} & 0.850 & 0.500 & \multicolumn{2}{|c|}{0.878} \\
\hline Surface & All & 0.1 & 29.7 & 0.67 & 0.38 & 0.09 & 0.79 & 0.67 \\
\hline Fit & $\mathrm{R}^{2}$ & -3.45 & \multicolumn{2}{|c|}{0.861} & 0.570 & 0.729 & \multicolumn{2}{|c|}{0.861} \\
\hline Buried & All & 0.1 & & & & & & \\
\hline Fit & $\mathrm{R}^{2}$ & 0.807 & & & & & & \\
\hline \multirow[t]{4}{*}{ US-131 } & Buried & 0.6 & 54.75 & 0.76 & 0.93 & 0.196 & 0.93 & 0.76 \\
\hline & $\mathrm{R}^{2}$ & 0.572 & \multicolumn{2}{|c|}{0.826} & 0.522 & 0.740 & \multicolumn{2}{|c|}{0.826} \\
\hline & Surface & 0.2 & $1.16 \mathrm{E} 7$ & 5.81 & 4.61 & 0.52 & $1.87 \mathrm{E}-5$ & 4.48 \\
\hline & $\mathrm{R}^{2}$ & 0.574 & \multicolumn{2}{|c|}{0.809} & 0.784 & 0.355 & \multicolumn{2}{|c|}{0.728} \\
\hline Surface & All & 0.1 & 55 & 0.76 & 0.52 & 0.198 & 0.95 & 0.76 \\
\hline Fit & $\mathrm{R}^{2}$ & -1.637 & \multicolumn{2}{|c|}{0.884} & 0.775 & 0.825 & \multicolumn{2}{|c|}{0.825} \\
\hline Buried & All & 0.1 & & & & & & \\
\hline Fit & $\mathrm{R}^{2}$ & 0.849 & & & & & & \\
\hline
\end{tabular}

\section{CONCLUSIONS}

Vibration attenuation data recorded in the near field with buried sensors and the far field with a conventional line array of surface geophones at two sites is presented. Attenuation relationships for pile driving induced vibrations were fitted through the data and trends were examined. Higher attenuation parameters were calculated when the pile was being driven in a clay deposit than in a sandy layer. This variation is expected, as attenuation rates are soil-specific (Woods and Jedele 1985) and vibrations tend to attenuate faster through softer materials. Empirical correlations are easy to implement and valuable to the piling practitioner, however based on the analysis presented here, can underestimate or overestimate the expected ground vibrations. The Bornitz equation was found to be a satisfactory correlation for both sand and clay materials and is recommended to be used as a preliminary evaluation to determine if pile driving induced vibrations 
would create potential problems. It is acknowledged however, that attenuation through the soil involves multiple reflections and refractions caused by soil layering which are not depicted by the Bornitz formula. Thus, field data collection, especially for critical projects, is necessary to verify if a prediction model fits well the monitored data. If recorded data are not available, then soildependent attenuation parameters (e.g. Table 2) may be utilized.

\section{ACKNOWLEDGMENTS}

The work described here was funded by the Michigan Department of Transportation, project \#114128, contract 2010-0296.

\section{REFERENCES}

Athanasopoulos-Zekkos, A., Woods, R. D. and Grizi, A. (2013). "Effect of pile-driving induced vibrations on nearby structures and other assets", Final Report to Michigan Department of Transportation, ORBP Number OR10-046, No. RC-1600.

Attewell, P. B. and Farmer, I. W. (1973). “Attenuation of Ground Vibrations from Pile Driving", Ground Engineering, Vol. 3, No. 7, pp. 26-29.

Attewell, P. B., Selby, A. R. and O'Donnell, L. (1992a). "Estimation of ground vibration from driven piling based on statistical analyses of recorded data", Geotechnical and Geological Engineering, Vol. 10, No. 1, pp. 41-59.

Attewell, P. B., Selby, A. R. and O'Donnell, L. (1992b). "Tables and graphs for the estimation of ground vibration from driven piling operations", Geotechnical and Geological Engineering, Vol. 10, No. 1, pp. 61-85.

Bornitz, G. (1931). "Über die Ausbreitung der von Großkolbenmaschinen erzeugten Bodenschwingungen in die Tiefe", J. Springer, Berlin (In German).

Dowding, C. H. (1996). “Construction Vibrations", Prentice-Hall, NJ.

Gkrizi, A. (2017). "Characterization of Pile Driving Induced Ground Motions", Ph.D. Thesis, Department of Civil and Environmental Engineering, University of Michigan.

Gutowski, T. G. and Dym, C. L. (1976). "Propagation of ground vibration: A Review", Journal of Sound and Vibration, Vol. 49, No. 2, pp.179-193.

Heckman, W. S. and Hagerty, D. J. (1978). "Vibrations associated with pile driving", Journal of the Construction Division, ASCE, Vol. 104, No. CO4, pp. 385-394.

Hendriks, R. (2004). "Transportation related earthborne vibrations (Caltrans Experiences)", California Department of Transportation, Technical Advisory, TAV-02-01-R9601.

Wiss, J. F. (1967). "Damage Effects of Pile Driving Vibrations", Highway Research Board Record No. 155 , pp. 14-20.

Wiss, J. F. (1981). "Construction vibrations: State-of-the-Art", Journal of Geotechnical and Geoenvironmental Engineering Division, ASCE, Vol. 107, No. GT2, pp. 167-181.

Woods, R. D., and Jedele, L. P. (1985). "Energy-Attenuation Relationships from Construction Vibrations", Vibration problems in geotechnical engineering, ASCE, Proceedings of a symposium sponsored by the Geotechnical Engineering Division, Detroit, Michigan, pp. 229-246. 